Ann. Zootech., I975, 24 (3), 559-563.

NOTE

\title{
NOTE SUR L'ESTIMATION \\ DE LA PRODUCTION LAITIÈRE DES VACHES ALLAITANTES AU DÉBUT DE LA LACTATION
}

\author{
P. LE NEINDRE ct M. PETIT \\ avec la collaboration technique de H. DubroeucQ \\ Laboratoive de la Production de Viande, \\ Centre de Recherches de Clermont-Ferrand, I. N. R. A., \\ Theix, Saint Genès Champanelle, 63110 Beaumont

\section{RÉSUMÉ}

Nous avons comparé chez des vaches allaitantes trois méthodes de mesure de la production laitière au début de la lactation (deux premiers mois) :

- des pesées avant et après la tétée (toutes les $5 \mathrm{~h}$ ) qui correspondent à peu près au rythme naturel des tétées chez le jeune veau;

- des pesées avant et après la tétée deux feis en 24 heures;

- la traite après injection d'ocytocine.

La première méthode apparaît peu précise et peu fiable.

Il est préférable d'utiliser l'une des deux autres méthodes bien qu'elles soient moins précises à cet âge que par la suite.

\section{INTRODUCTION}

Dans une première étude (LE NEINDRE, 1973) effectuée à partir du second mois de lactation, nous avons montré que la pesée du veau avant et après la tétée, deux fois par jour, donne une estimation satisfaisante de la production laitière des vaches allaitantes au-delà du second mois de lactation. En effet, la variabilité de la mesure est faible d'un jour à l'autre et diminue avec l'avancement de la lactation (coefficient de variation de 7.7 à $5.7 \mathrm{p}$. Ioo). Il en est de même pour la quantité de lait résiduel après la tétée estimée par le lait trait après une injection intraveineuse de 5 UI d'ocytocine. En deux tétées, le veau âgé de plus de 2 mois parait donc capable de boire tout le lait produit par sa mère en 24 heures. 
Cependant, les veaux âgés de moins de 2 mois, tètent fréquemment $(6,0 \pm 3,0$ tétées/j) quand ils sont en liberté avec lcur mère ; réduire à deux le nombre de tétées journalières risque de faire sous-estimer la quantité de lait bue habituellement. Dans la présente étude, nous avons estimé, au début de la lactation, la production laitière de vaches en liberté avec leurs veaux; le jour de la mesure, les veaux tétaient sous surveillance à intervalle de 5 à 7 heures et étaient pesés avant et après trois des quatre tétées journalières ( ${ }^{r e}$ méthode). Nous avons comparé cette première estimation à celles obtenues par deux autres méthodes couramment utilisées : la pesée du veau avant et après la tétée deux fois en 24 heures ( $2^{\mathrm{e}}$ méthode) et la traite du lait sécrété entre deux injections d'ocytocine espacées, de 5 heures ( $3^{\mathrm{e}}$ méthode).

\section{MATÉRIEL E'T MÉTHODES}

Les animaux sont les mêmes que ccux utilisés dans une étude sur le comportement précédent (P. LE Neindre et M. Ptetr, I975). La production laitière a été estimée une fois par semaine du vêlage jusqu'au troisième mois. Nous avons comparé, alternativement une semaine sur deux, la première méthode à la deuxième, puis à la troisième méthode.

Chaque semaine, les veaux étaient séparés de leur mère le matin vers 7 heures et tétaient sous surveillance à $I 2$ heures. Ils étaient pesés avant et après la tétée (à 0,1 kg près) à 17,22 heures et à 5 heures le lendemain matin (fig. I).

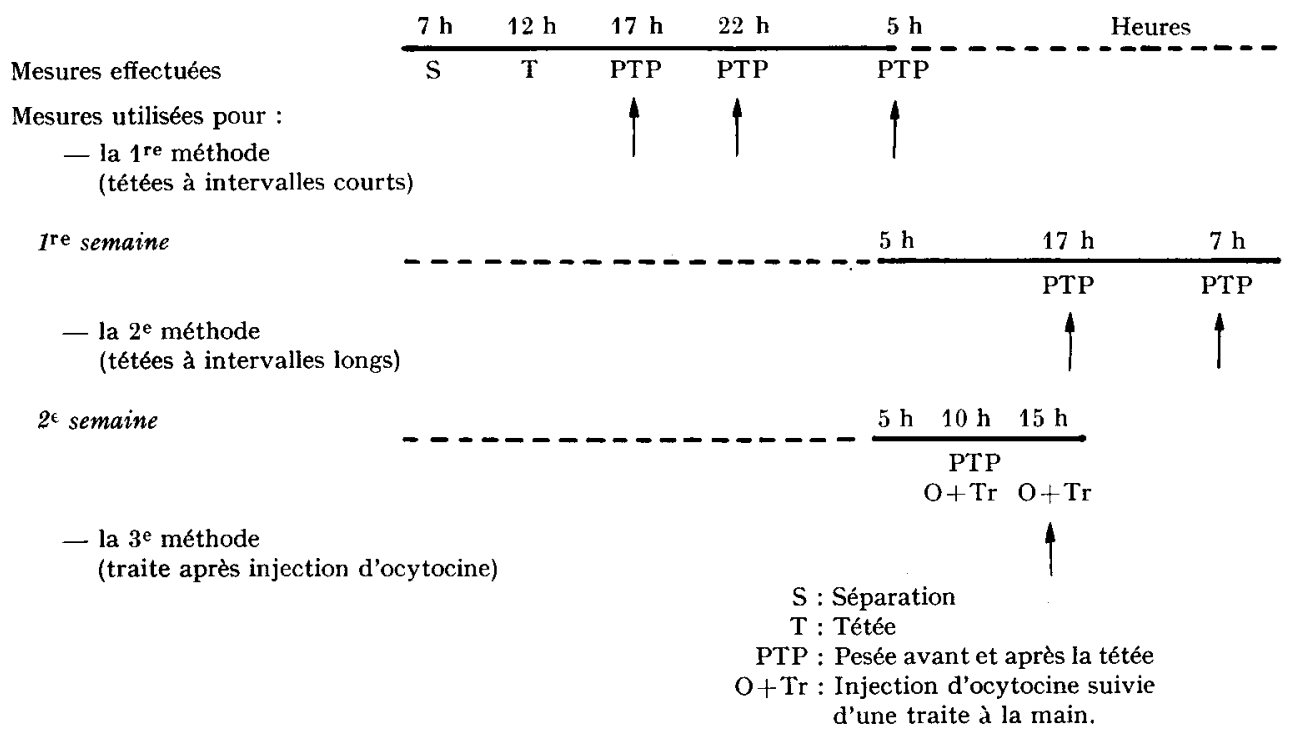

FIG. I. - Mesures utilisées pour comparer les diffèrentes estimations de la production laitière

Une semaine sur deux, les veaux étaient pesés le second jour avant ct après la tétéc le soir à $\mathbf{} 7$ heures et le lendemain matin à 7 heures; la quantité de lait bue à ces deux tétées a donné l'estimation de la production laitière à partir des tétées à intervalles longs.

L'autre semaine, ils tétaient de nouveau à so heures et le lait restant dans la mamelle était trait à la main après une injection intraveineuse d'ocytocine (5 UI, Syntocinon); 5 heures après environ, une nouvelle traite après injection d'ocytocine permettait d'estimer la production lai- 
tière dans l'intervalle. Ces injections d'ocytocine étaient faites à l'aide d'un cathéter fixé dans une veine jugulaire quelques heures avant les mesures.

Nous avons noté de façon précise les horaires de mesures. Cela nous a permis, pour chacune des trois méthodes, de calculer une production laitière horaire puis journalière.

\section{RÉSULTATS ET DISCUSSION}

\section{I. - Comparaison entre les différentes méthodes d'estimation}

Les données moyennes obtenues à partir des trois méthodes ne sont pas significativement différentes. En revanche, les variances plus élevées pour la première semblent indiquer que celle-ci est la moins précise. Cette imprécision est certainement due à la multiplication des pesées $(6$ pesées pour une estimation), mais également à une perturbation plus importante des veaux qui assez souvent refusaient de téter à horaires réguliers et fixés (la consommation par tétée a été inférieure à $0,5 \mathrm{~kg}$ dans I I p. Ioo des cas).

Les corrélations entre la première méthode ct les deux autres sont faibles, et significatives seulement avec la deuxième méthode (tabl. I).

TABLEAU I

Liaisons entre les estimations

\begin{tabular}{|c|c|c|c|}
\hline & & Production laitière $(\mathrm{kg})$ & Corrélation \\
\hline $\begin{array}{c}1^{\mathrm{e}} \text { comparaison } \\
\text { (29 couples) }\end{array}$ & Tétée à intervalle court & $6,75 \pm 5,35$ & \multirow{2}{*}{$r=0,55^{*}$} \\
\hline $\begin{array}{l}\text { Age des veaux : } \\
\qquad 37,3 \pm 21,1 \text { jours }\end{array}$ & Tétée à intervalle long & $7,24 \pm 1,96$ & \\
\hline $\begin{array}{l}2 \text { e comparaison } \\
\text { (35 couples) }\end{array}$ & Tétée à intervalle court & $7,14 \pm 3,97$ & \multirow{2}{*}{$r=0,35$} \\
\hline $\begin{array}{l}\text { Age des veaux : } \\
\qquad 45,9 \pm 17,6 \text { jours }\end{array}$ & $\begin{array}{l}\text { Traite après injection } \\
\text { d'ocy tocine }\end{array}$ & $7,34 \pm 2,86$ & \\
\hline
\end{tabular}

* Significatif à 5 p. Ioo.

La variabilité de la quantité de lait bue lors d'une tétée est beaucoup plus importante avec les tétées à intervalles courts qu'avec celles à intervalles longs; les coefficients de variation, quand l'influence de l'heure de la tétée et du jour sont éliminés, sont respectivement de 48,8 p. roo et 16,7 p. IOO. De plus, quand l'intervalle entre tétées est court ( ${ }^{\text {re }}$ méthode). les veaux tètent de façon différente suivant l'heure.

\section{2. -- Quantités de lait résiduel}

Les quantités de lait restant dans la mamelle après la tétée de ro heures sont très variables (fig. 2). Elles décroissent rapidement lorsque s'accroît le stade de lactation, bien que les quantités de lait bues à la tétée n'augmentent pas. Les pourcentages de lait résiduel par rapport au lait total sont identiques pour les deux races de vaches, malgré leurs productions laitières très différentes (tabl. 2). 




FIG. 2. - Estimations de la quantité de lait restant dans la mamelle après la tétée du veau

\section{TABLEAU 2}

Infuence du stade de lactation et du niveau de production laitière sur les quantités de lait résiduel

\begin{tabular}{|c|c|c|c|c|c|}
\hline & \multirow[b]{2}{*}{$\begin{array}{c}\text { Nombre } \\
\text { de mesures }\end{array}$} & \multirow[b]{2}{*}{$\begin{array}{c}\text { Age } \\
\text { des veaux }(\mathbf{j})\end{array}$} & \multirow{2}{*}{$\begin{array}{l}\text { Product. laitière } \\
\text { journalière }(\mathrm{kg})\end{array}$} & \multicolumn{2}{|c|}{ Tétée de $10 \mathrm{~h}$} \\
\hline & & & & Lait bu (kg) & $\begin{array}{c}\text { Lait résiduel } \\
(\mathrm{kg})\end{array}$ \\
\hline \multirow[b]{2}{*}{ Charolaise } & \multicolumn{5}{|c|}{ Age des veaux $<50$ jours } \\
\hline & 11 & $27,8 \pm 13,7$ & $5,90 \pm 1,73$ & $1,50 \pm 1,2 / \mathbf{t}$ & $1,37 \pm 1,10$ \\
\hline \multirow[t]{2}{*}{ Salers } & 13 & $20,85 \pm 11,05$ & $7,73 \pm 1,56$ & $2,14 \pm 0,87$ & $2,18 \pm 2,0^{\prime}$ \\
\hline & \multicolumn{5}{|c|}{ Age des veaux $>50$ jours } \\
\hline Charolaise & 6 & $73,6 \pm 13,4$ & $6,21 \pm \pm 2,66$ & $1,03 \pm 0,63$ & $0,57 \pm 0,29$ \\
\hline Salers & 6 & 61,0 土 10,4 & $7,90 \pm 1,54$ & $2,1 \pm 0,83$ & $1,04 \pm 0,34$ \\
\hline
\end{tabular}

\section{CONCL,USION}

La méthode utilisant les tétées à intervalles courts que nous supposions à priori la meilleure, est très peu fiable mais donne des estimations moyennes de la production laitière identiques à celles des autres méthodes, contrairement à ce qu'obtenait CHow et al. (1967).

Il semble donc préférable d'utiliser l'une des deux autres méthodes, la pesée avant et après la tétée 2 fois en 24 heures ou la traite après injection d'ocytocine; cependant, les résultats sont beaucoup plus variables en début de lactation que par la suite. 


\author{
SUMMARY \\ ESTIMATION OF MILK PRODUCTION \\ IN NURSING COWS DURING EARLY LACTATION
}

Three methods for accessing the milk production of nursing cows during the first two months of lactation, were compared. The methods used were the following :

- weighing of the calves before and after suckling at five hours intervals. This approximately corresponded to the natural suckling behaviour of the young calves;

- weighing of the calves before and after suckling twice per 24 hours;

- hand milking after an injection of oxytocin (5 IU);

The first method is not precise enough. The two others are more advisable clespitc the fact that they are less accurate at this stage of lactation than at later stages.

\title{
RÉFÉRENCES BIBLIOGRAPHIQUES
}

Chow H., Riggs J. K., Schake L. M., I967. Frequency of milking and milk yield of beef cows. J. Anim. Sci., 26, 202 (Abstr. no 5).

Le Neindre P., I973. Observations sur l'estimation de la production laitière des vaches allaitantes. Ann. Zootech., 22, 413-422.

Le Neindre P., Petit M., I975. Nombre de tétées et temps de pâturage des veaux dans les troupeaux de vaches allaitantes. Ann. Zootech, 24, 553-558. 